\title{
Estudio de los efectos en los sistemas de iluminación electrónica en función de la calidad de la energía en los sistemas automotrices
}

\section{Study of the effects on electronic lighting systems based on energy quality in automotive systems}

\author{
MENDOZA-RIVERA, José†*, JUAREZ-BALDERAS, Mario Alberto, LOPEZ-NUÑEZ Adolfo Rafael, \\ VAZQUEZ-GUZMAN, Gerardo
}

Instituto Tecnológico Superior de Irapuato

ID $1^{\mathrm{er}}$ Autor: José, Mendoza-Rivera / ORC ID: 0000-0002-8178-1296

ID $1^{\text {er }}$ Coautor: Mario Alberto, Juarez-Balderas / ORC ID: 0000-0002-5756-5403

ID $2^{\text {do }}$ Coautor: Adolfo Rafael, Nunez-Lopez / ORC ID: 0000-0003-3116-5150

ID $3^{\text {er }}$ Coautor: Gerardo, Vázquez-Guzman / ORC ID: 0000-0002-9915-1161

DOI: 10.35429/JID.2019.7.3.14.19

Recibido 09 Marzo, 2019; Aceptado 30 de Junio, 2019

\begin{abstract}
Resumen
Actualmente los automóviles a combustión poseen un amplio número de equipos electrónicos: la unidad de control electrónico, sensores de posición, de flujo, sistema de freno automático, sistema ABS, entre otros. Actualmente y en grandes rasgos los automóviles un conjunto de partes mecánicas gobernadas por un sistema electrónico. Se plantea a futuro los automóviles sean electrónicos en su totalidad, pero anterior a este paso se desarrollarán los automóviles híbridos los cuales tiene sus respectivos motores eléctricos controlados por una etapa de potencia y su motor de combustión interna (Cevallos 2016). En el presenta trabajo se identifican las perturbaciones en los sistemas automotrices que se introducen a la micro red del automóvil, sus efectos para analizar los efectos que pueden tener en el sistema de iluminación de tipo LED. Objetivo: Realizar un estudio de las perturbaciones (transitorias y permanentes) en sistemas automotrices. Metodología: Para trabajo propuesto se llevó a abordaron los siguientes puntos: Antecedentes sobre perturbaciones automotrices. Origen y comportamiento típico de las señales continuas Estudio y análisis de los componentes que introducen estas perturbaciones transitorias. Se cuantifican las señales de los sistemas que introducen perturbaciones a la red de $\mathrm{CD}$ en los automóviles en un simulador de señales eléctricas en este caso PSpice $\odot$, así como la aplicación de la transformada Rápida de Fourier (FFT). Contribución: Al análisis de perturbaciones en automóviles e identificar los posibles fallos que pueden existir a causa de estos.
\end{abstract}

Batería, Armónicos, Perturbaciones

\begin{abstract}
Currently, the combustion cars of a large number of electronic equipment: the electronic control unit, position sensors, flow sensors, automatic brake system, ABS system, among others. Currently and in broad strokes cars a set of mechanical parts controlled by an electronic system. In the future, cars are fully electronic, but prior to this step, hybrid cars will be developed which have their respective electric motors controlled by a power stage and their internal combustion engine (Cevallos 2016). This paper identifies the disturbances in the automotive systems that are introduced to the micro network of the automobile, its effects to analyze the effects that may have on the LED lighting system. Objetivos: Estudiar los transitorios y permanentes en automóviles para clasificar su comportamiento. Analizar las perturbaciones que pudieran ser perjudiciales para los sistemas electrónicos del automóvil. Cuantificar de perturbaciones a partir de la Transformada Rápida de Fourier (FFT). Estudio y análisis de los efectos a través de la simulación numérica de los transitorios en convertidos de CD-CD empleado para la iluminación.
\end{abstract}

Battery, Harmonics, Automobile

Citación: MENDOZA-RIVERA, José, JUAREZ-BALDERAS, Mario Alberto, LOPEZ-NUÑEZ Adolfo Rafael, VAZQUEZGUZMAN, Gerardo. Estudio de los efectos en los sistemas de iluminación electrónica en función de la calidad de la energía en los sistemas automotrices. Revista del Diseño Innovativo. 2019. 3-7: 14-19

\footnotetext{
* Correspondencia del Autor (Correo electrónico: mario.juarez@itesi.edu.mx)

$\dagger$ Investigador contribuyendo como primer autor.
} 


\section{Introducción}

Los sistemas eléctricos propios del automóvil generan perturbaciones eléctricas debido a los dispositivos inductivos automóvil tales como el alternador, ignitor, carga de batería, etc, dichas perturbaciones anteriormente no eran tomadas en cuenta y los sistemas electrónicos entraban en funcionamiento después del transitorio inicial. Las perturbaciones generadas pueden ocasionar desde mal funcionamiento en los sistemas eléctricos y electrónicos del automóvil hasta un daño permanente a los sistemas. (Mancada 2013).

Entre las principales perturbaciones del sistema son las que se presentan en la carga y descarga de la batería donde tiene presencia el alternador. La señal de carga tiene un rizo el cual depende de dos factores, a) la corriente generada por el alternador $\mathrm{y} b$ ) las revoluciones a las que el alternador se encuentra sometido. Este rizo contiene armónicos por la rectificación realizada por diodos internos del alternador.

A continuación, se presenta un esquema general de la red de $\mathrm{CD}$ de un automóvil convencional (Fig. 1), en donde se muestran los circuitos que inyectan perturbaciones en la red (Pollock 2015),(Robyn 2007).

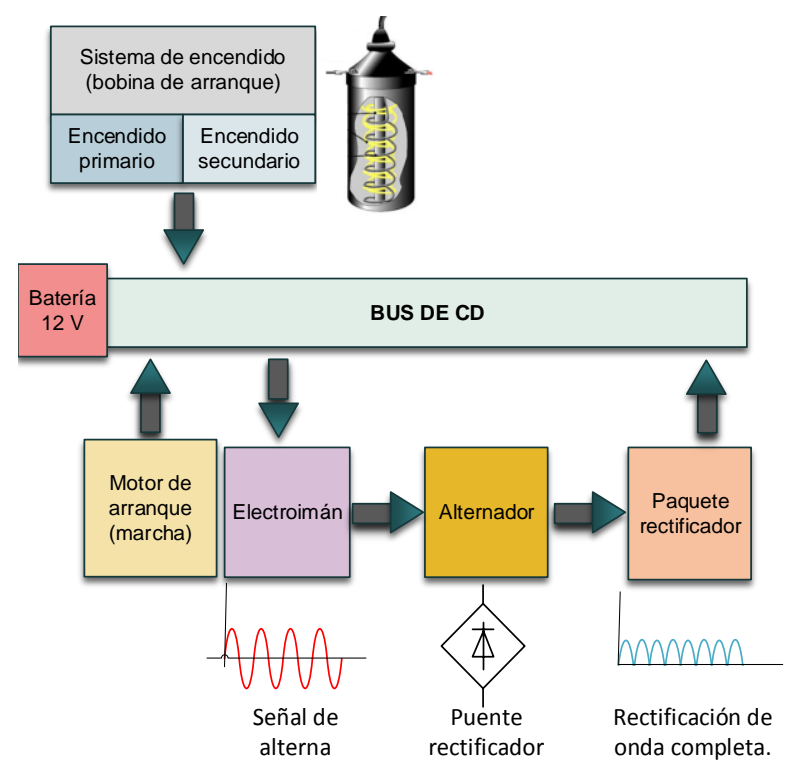

Figura 1 Sistemas que introducen perturbaciones a la red de DC en automóviles convencionales

Con el fin de analizar los efectos en los sistemas de iluminación electrónica, existe la necesidad de interpretar el funcionamiento y comportamiento de los sistemas que introducen perturbaciones al BUS de CD un automóvil.
Ese modelado interpreta su funcionamiento a partir de resistencias, capacitores e inductores para aplicar las diferentes técnicas de análisis.

El primer paso consiste en modelar la batería, ya que es necesario modelar el componente encargado el suministro y almacenamiento de energía. En la Fig. 3 se muestra el circuito eléctrico equivalente para una celda de una batería acido plomo (Robyn 2007). Por otro lado, se ha descartado el análisis del motor de arranque el cual solo presenta un sobre tiro de corriente al iniciar el motor de combustión interna., uno del requerimiento del análisis consiste en que una señal sea repetitiva o períoca para cuantificar su nivel de contaminación.

Segundo paso consiste en modelar el sistema de carga como se muestra en la Fig. 4 El cual es el encargado de suministrar la mayoría de energía necesaria una vez encendido el automóvil.

El tercer paso se encentra en analizar el sistema de ignición mostrado en la Fig. 7 el cual realiza la quema de aire-combustible requerida para el funcionamiento de motor de combustión interna.

Por último, es cuantificar cada uno de los sistemas y obtener estas señales de perturbación para introducirlas al controlador de cargas LED' el cual es el sistema requerido para el suministro necesario de las cargas LED's, con el fin de ver los efectos que estas tienen así mismo evaluar la introducción de un convertidor CD-CD entre los sistemas que introducen las perturbaciones y el controlador de cargas LED' como se muestra gráficamente en la Fig. 2.

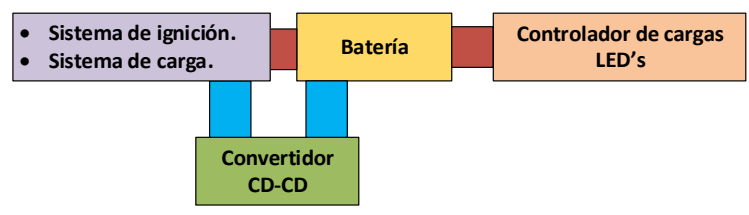

Figura 2 Plan de análisis y propuesta de diseño

\section{Desarrollo}

Las simulaciones del sistema eléctrico dependen en gran medida del submodelo de la batería, que es el componente más complejo de simular. Los métodos para modelar la batería son generalmente laboriosos y difíciles (Robyn 2007).

MENDOZA-RIVERA, José, JUAREZ-BALDERAS, Mario Alberto, LOPEZ-NUÑEZ Adolfo Rafael, VAZQUEZ-GUZMAN, Gerardo. Estudio de los efectos en los sistemas de iluminación electrónica en función de la calidad de la energía en los sistemas automotrices. Revista del Diseño Innovativo. 2019 


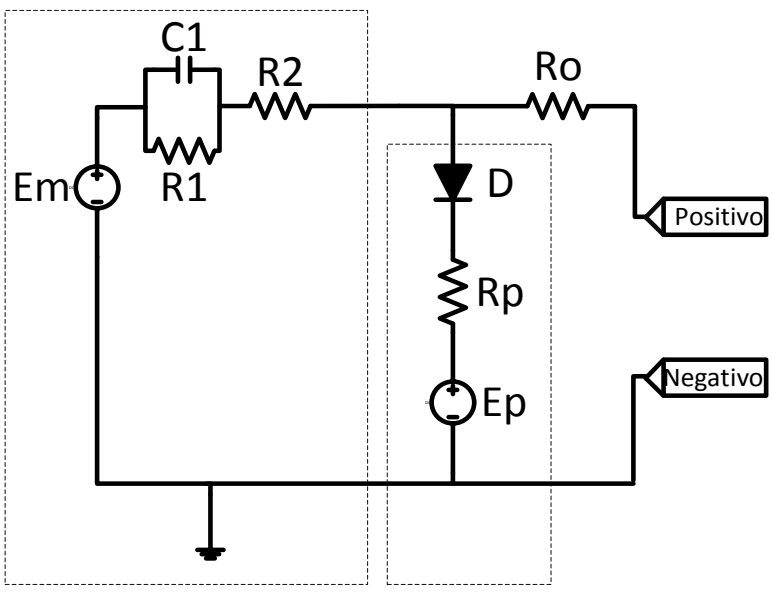

Figura 3 Circuito eléctrico equivalente de una celda de una batería acido plomo

Las ecuaciones para determinar el valor de los componentes eléctricos para el circuito de la figura 2 se describen el artículo de (Robyn A. 2007). Donde los parámetros son determinados a partir de factores influentes como estado de carga de la batería inicial, temperatura ambiente, temperatura del electrolito, voltaje en circuito abierto.

Un factor importante sucede cuando el estado de carga de la batería es alto, se presenta una corriente parásita mostrada en la Fig. 3, la cual tiene dependencia con la temperatura del electrolito y del voltaje en la rama parásita.

La ecuación 1 se aproxima a la fuerza electromotora interna (Em), o al voltaje de circuito abierto de una celda (Robyn A. 2007).

$E_{m}=E_{m 0}-K_{E}(273+\theta)(1-S O C)$

Donde:

$E_{m}=$ Tensión de circuito abierto (EMF).

$E_{m 0}=$ Tensión de circuito abierto a plena carga.

$K_{E}=$ Constante en voltios $/{ }^{\circ} \mathrm{C}$.

$\theta=$ Temperatura del electrolito en ${ }^{\circ} \mathrm{C}$.

$\mathrm{SOC}=$ estado de carga de la batería.

Para este artículo se asumen que el valor de "Em" es constante cuando la batería está completamente cargada. La Em varía con la temperatura y el estado de carga (SOC), en este caso se toma una temperatura constante y un estado de carga constante.

a) Resistencia en las terminales: impedancia constante entre las terminales de la batería, la cual se asume que todas las temperaturas son constantes. b) Resistencia de la rama principal $\left(\mathrm{R}_{1}\right)$ : varía con la profundidad de la carga (DOC) y es una medida de la carga de la batería ajustada para la corriente de descarga. La resistencia aumentó exponencialmente a medida que la batería se agotaba durante una descarga.

c) Capacitancia de la rama principal $\left(\mathrm{C}_{1}\right)$ : Denota un retraso de voltaje cuando cambia la corriente de la batería.

d) Resistencia serie de la rama principal $\left(\mathrm{R}_{2}\right)$ : La resistencia aumenta exponencialmente a medida que aumenta el estado de carga de la batería. La resistencia también varía con la corriente que fluye a través de la rama principal. Esta resistencia tiene especialmente influencia en la batería durante la carga. La resistencia se vuelve relativamente insignificante para las corrientes de descarga.

e) Corriente en la rama parasita $\left(\mathrm{i}_{\mathrm{p}}\right)$ : ocurre cuando la batería se está cargando. La corriente depende de la temperatura del electrolito y del voltaje en la rama parásita. La corriente es pequeña en la mayoría de las condiciones, excepto durante la carga en SOC.

\section{Señales de carga del alternador a la betería}

Se utilizó el modelado una batería de ácido plomo con características como $12 \mathrm{~V}$ de en circuito abierto, capacidad de $50 \mathrm{Ah}$, a una temperatura ambiente con un estado de carga de la batería (SOC) a 0.8 los valores de los elementos se enlistan en la Tabla 1. Así mismo se utilizó el modelo de un alternador A-115R el cual tiene una capacidad de suministro máximo de 55 Amperios a 6000 RMP [7]. El alternador A-115R utiliza un regulador de tensión RT-7 que tiene buena estabilidad térmica y un rango amplio de temperatura de trabajo [7].

\begin{tabular}{|l|r|}
\hline Elemento & \multicolumn{1}{c|}{ Valor } \\
\hline C & $621.3 \mathrm{uF}$ \\
\hline R1 & $0.001609 \Omega$ \\
\hline R2 & $0.0015 \Omega$ \\
\hline Ro & $3.696^{*} 10^{\wedge}-3 \Omega$ \\
\hline Rp & 0.02 \\
\hline Em & $2.111 \mathrm{~V}$ \\
\hline Ep & $1.95 \mathrm{~V}$ \\
\hline
\end{tabular}

Tabla 1 Valores usados para cada celda de la batería

MENDOZA-RIVERA, José, JUAREZ-BALDERAS, Mario Alberto, LOPEZ-NUÑEZ Adolfo Rafael, VAZQUEZ-GUZMAN, Gerardo. Estudio de los efectos en los sistemas de iluminación electrónica en función de la calidad de la energía en los sistemas automotrices. Revista del Diseño Innovativo. 2019 
En la figura 3 se muestra el diagrama de conexión del sistema de carga de la batería. El cual consta de tres devanados conectado en estrella, contiene seis diodos, de los cuales seis son usados para la rectificación de la señal en cada devanado,

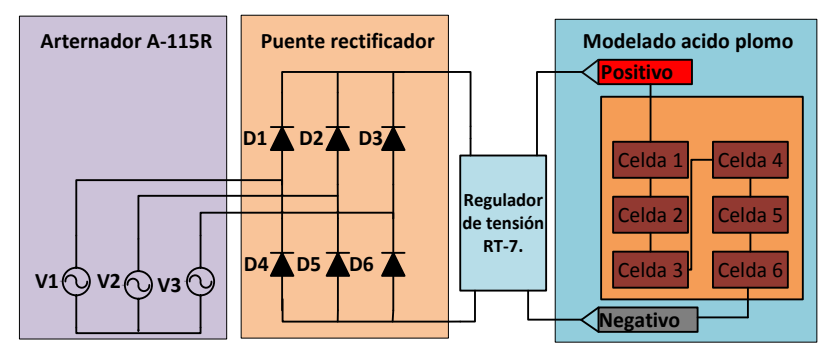

Figura 3 Sistema de carga de la batería a 6000 rpm $=100$ $\mathrm{Hz}$

En la Fig. 4 se presenta las señales en el alternador $\mathrm{V} 1, \mathrm{~V} 2, \mathrm{~V} 3$ así mismo las señales entre línea V11, V12 y V13 los cuales están desfasados $30^{\circ}$ con respecto a las tensiones de cada devanado del alternador.

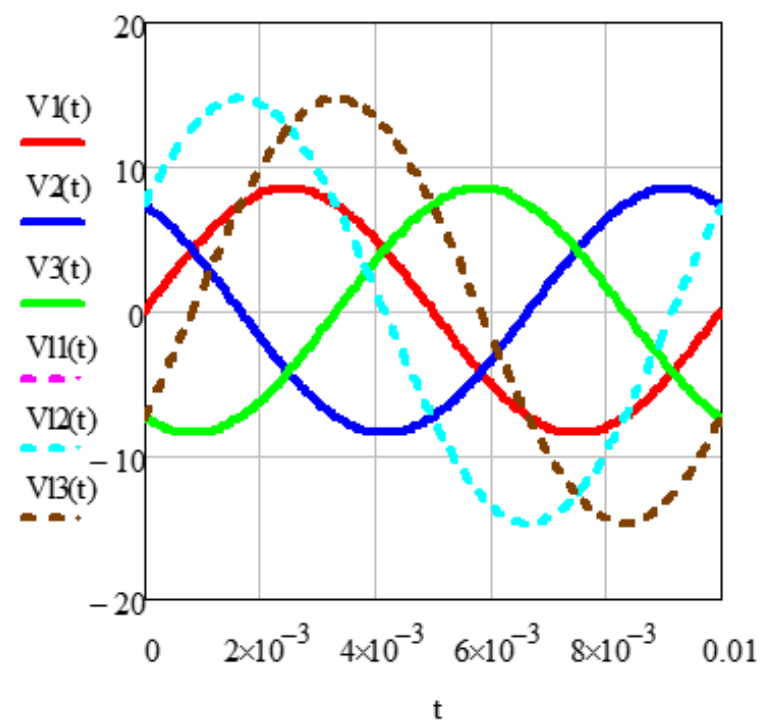

Figura 4 Tensiones del alternador V1, V2 y V3 y tensiones de línea V11, Vl2 y V13

En la Figura 5 se muestra el rizo proveniente del alternador, conectado a la batería el cual tiene una forma pulsante la cual oscila a 2 Vpp aproximadamente, el cual es el resultado de la rectificación de los diodos ha aumentado en 6 veces la frecuencia natural.

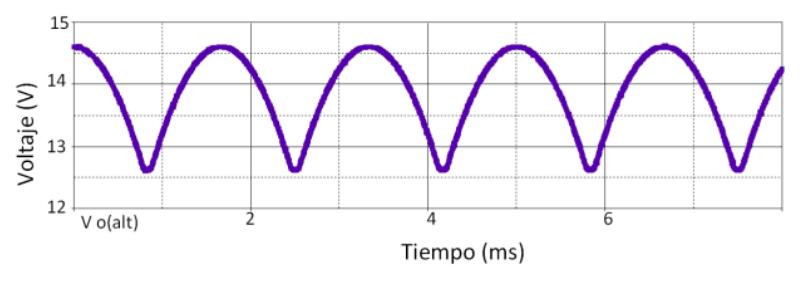

Figura 5 Rizo del sistema de carga a $600 \mathrm{~Hz}$
En la Tabla 2 se presentan los datos importantes de la del rizo del sistema de carga.

\begin{tabular}{|l|r|}
\hline Datos & \multicolumn{1}{|l|}{ Valor } \\
\hline Tensión promedio (Vprom) & $14.059 \mathrm{~V}$ \\
\hline Corriente promedio (Iprom) & $0.469 \mathrm{~A}$ \\
\hline Voltaje RMS (VRMS) & $14.071 \mathrm{~V}$ \\
\hline Corriente RMS (IRMS) & $0.469 \mathrm{~A}$ \\
\hline Potencia promedio de salida (Pprom) & $6.588 \mathrm{~W}$ \\
\hline Potencia RMS de salida (PRMS) & $6.6 \mathrm{~W}$ \\
\hline Factor de forma (FF) & 1.001 \\
\hline Factor de cresta (FC) & 0.604 \\
\hline Factor de rizo (FR) & 0.042 \\
\hline
\end{tabular}

Tabla 2 Datos importantes del rizo de sistema de carga

Se tiene como resultada un factor de forma de 1.001 el cual es el cociente del valor RMS y el valor promedio de la tensión del rizo, por otro lado, el factor de cresta es de 0.604 el cual es el valor pico de la señal sobre el valor RMS de la señal, el factor de rizo es de 0.042 .

En la Figura 6 se muestra la FFT del voltaje en simulación, en la cual se muestran múltiplos de $100 \mathrm{~Hz}$ que es la frecuencia natural, estos corresponden al sexto armónico de $600 \mathrm{~Hz}$, al doceavo armónico de $1200 \mathrm{~Hz}$ y el dieciochoavo armónico $1800 \mathrm{~Hz}$ y al vigésimo cuarto armónico $2400 \mathrm{~Hz}$.

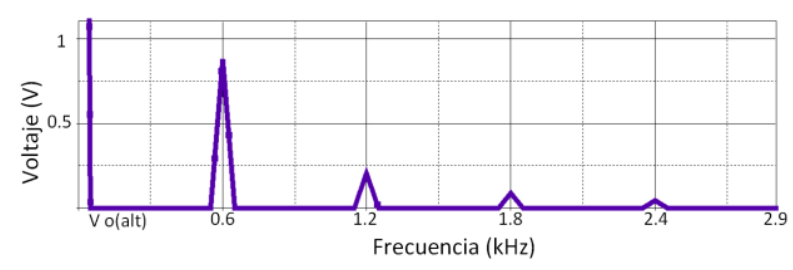

Figura 6 FFT del voltaje de carga del modelo completo de la batería a $6000 \mathrm{rpm}$

A continuación, en la Fig. 7 se muestra el análisis matemático de la señal del voltaje, donde se presenta el nivel de contaminación de sistema de carga de acuerdo con cada armónico, así como la distorsión armónica total (DAT) que corresponde a un valor de 3.557 . 


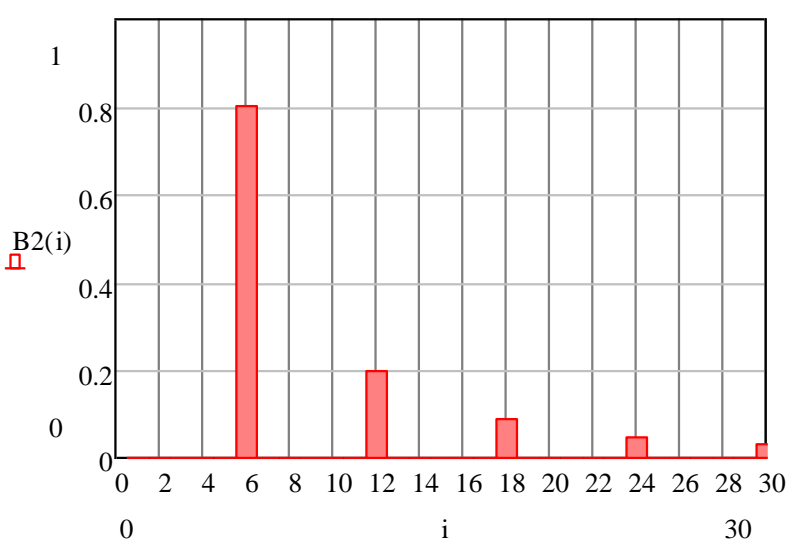

Figura 7 Nivel de contaminación para cada armónico

Se presenta una contaminación para el sexto armónico de $0.8 \mathrm{~V}$, para el doceavo de 0.2 $\mathrm{V}$, para el dieciochoavo de $0.1 \mathrm{~V}$ y $0.25 \mathrm{~V}$ para el vigésimo cuarto armónico.

\section{Señales del sistema de ignición}

A continuación, en la Fig. 8 se presenta circuito eléctrico el sistema de ignición el cual cuenta con un sistema de elevación de tensión con el fin de lograr el arco eléctrico en la bujía, basado en autotransformador del tipo Flyback (Bosch 2006).

Este nivel de tensión es logrado a partir de un sistema de autotransformador de bobinas acopladas donde Lp guarda un número relación de vueltas de 1:1000 aproximadamente con Ls (Bosch 2006). En la Figura 7 se presenta las señales del secundario de la bobina de arranque. Donde, el voltaje pico corresponde a una descarga de 3000 volts,

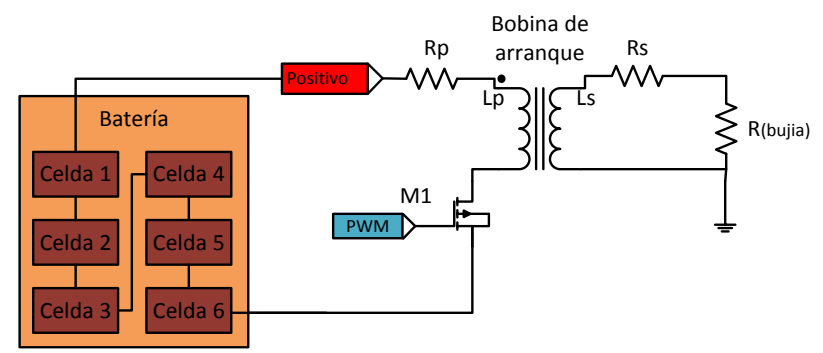

Figura 8 Circuito básico del sistema de ignición transistorizado

En la Fig. 9 se muestra las señales de salida del ignitor el cual corresponde a una salida de 3000V, en este ciclo el transformador almacena energía y la libera en el alto voltaje. Durante el ciclo de almacenamiento de energía la corriente de entrada del ignitor es pulsante y tiene una corriente pico de 98 Amps.

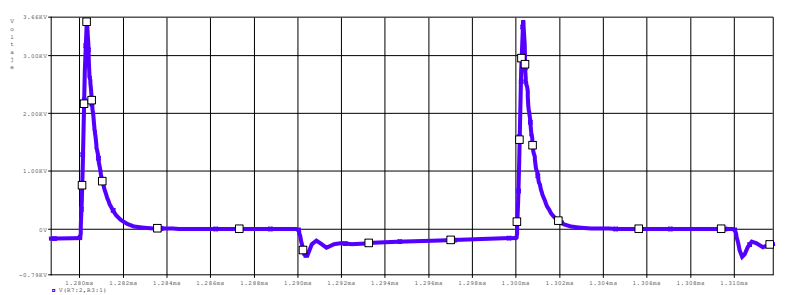

Figura 9 Voltaje a la salida del secundario del ignitor

En La Fig.10 se muestra la corriente de consumo del ignitor la cual se puede reflejar como distorsión en la batería.

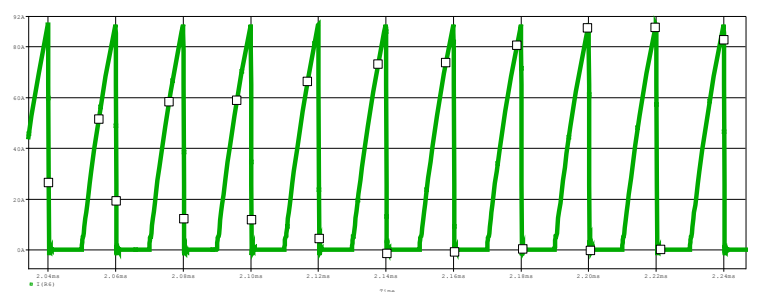

Figura 10 Corriente en la entrada del ignitor

\section{Efectos del convertidor CD-CD}

En la Fig. 11 se propone la introducción de un convertidor reductor CD-CD entre el sistema de carga y la batería para estudiar el efecto de las perturbaciones causadas por el sistema de carga.

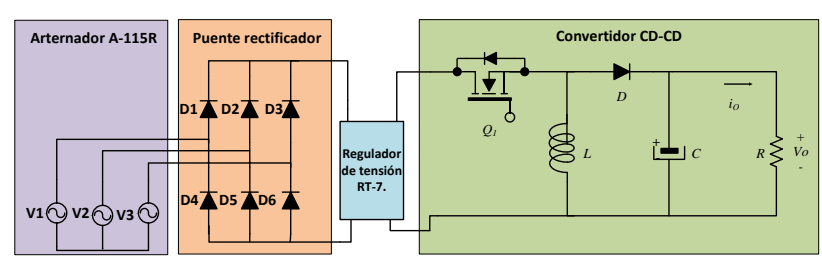

Figura 11 Propuesta de análisis

En la Figura 12-13 se realiza una comparativa entre los niveles de tensión y corriente en las entradas y salida del convertidor.

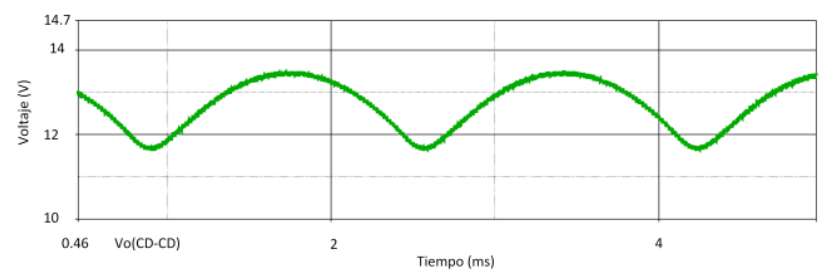

Figura 12 Corriente de entrada al convertidor CD-CD

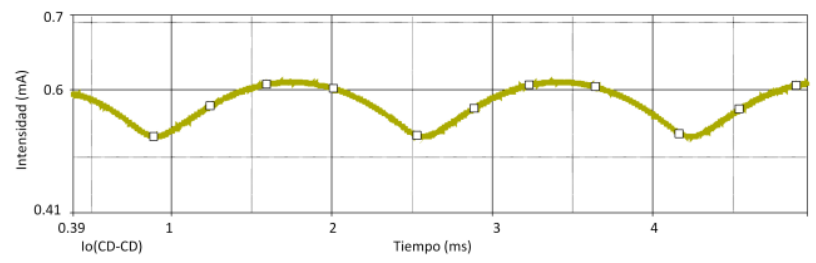

Figura 13 Corriente de salida del convertidor CD-CD

MENDOZA-RIVERA, José, JUAREZ-BALDERAS, Mario Alberto, LOPEZ-NUÑEZ Adolfo Rafael, VAZQUEZ-GUZMAN, Gerardo. Estudio de los efectos en los sistemas de iluminación electrónica en función de la calidad de la energía en los sistemas automotrices. Revista del Diseño Innovativo. 2019 
Al comparar el rizo de la Fig. 5 con el rizo de la Fig. 13 los cuales son el rizo del sistema de carga sin convertidor CD-CD y con convertidor CD-CD. Se presenta una disminución el nivel de tensión. Como se puede apreciar en las corrientes de entrada y salida la mayor parte de las perturbaciones corresponden a las ondulaciones de rectificación de alternador.

\section{Conclusión}

Después del análisis realizado al sistema de carga de batería el cual tiene un rizo de una frecuencia de $100 \mathrm{~Hz}$, el cual depende de la revoluciones y velocidad del motor, donde los armónicos característicos de dicha señal son el sexto, doceavo, dieciochoavo y vigésimo cuarto armónico, el rizo a la salida es el causante de la mayor contaminación de la red interna del automóvil, el caso del ignitor, la corriente de consumo, se ve poco reflejada en la red, y solo genera variaciones de voltaje, es importante considerar que para una nueva generación de automóviles, la rectificación y carga de batería debe emplearse convertidores electrónicos y de esta manera dichos convertidores podrán reducir el rizado de salida del bus de $\mathrm{CD}$, el autos electrónicos el bus de CD corresponde a 400V, lo que implica una proporción del rizado de salida menor.

\section{Referencias}

Alfonso Temístocles Cevallos Galarza. (2016). análisis de las señales del sistema de encendido primario y secundario de disparo para el módulo del vehículo Chevrolet aveo family 1.51 . Guayaquil. Ecuador: Universidad Internacional del Ecuador.

Anne Pollock; Helen Pollock; Charles Pollock. (2015). High Efficiency LED Power Supply. IEEE Journal of Emerging and Selected Topics in Power Electronics, 3, 617,623.

Christian Macanda. (2013). Perturbación en las redes de iluminación exterior y riesgos para los equipos (iluminación pública LED). Products \& Normalization Manager CITEL, 2, 18. 11 julio 2019, De Tecnorayo Base de datos.

Li Yulong, Wu Zhenjun. (2010). Statistical Analysis of the Disturbance Sources characteristics on Hybrid Electric Vehicle. IEEE, 1, 4. 12 Julio 2019, De IEEE Xplorer Base de datos.
Manuel Déleg. (2010). TECNOLOGÍA LED. Universidad Politécnica Salesiana Cuenca, Azuay Ecuador, 1, 4. 10 Julio 2019, De Monografías Base de datos.

Prestolite (2005) indiel motores de San Luis S. A. Manual de Alternadores.

Robert Bosch. (2006). Sistema de encendido. En BOSCH (1-7). Brasil: BOSCH.

Robyn A. Jackey . (2007). A Simple, Effective Lead-Acid Battery Modeling Process for Electrical System Component Selection. The MathWorks, 1,1 . 